\title{
CFD analysis of turbulent cross-flow in a staggered tube bundle equipped with grooved cylinders
}

\author{
O. Ladjedel ${ }^{1}$, L. Adjlout ${ }^{1}$, T.Yahiaoui ${ }^{2}$ and, O. Imine ${ }^{2}$ \\ ${ }^{1}$ laboratoire d'aérohydrodynamique naval Département de Génie Maritime Mechanical Engineering Faculty USTO Oran, \\ Oran 31000 Algeria \\ ${ }^{2}$ Laboratoire d'aéronautique et systèmes propulsive Département de Génie Mécanique Mechanical Engineering Faculty \\ USTO Oran, Oran 31000 Algeria
}

\begin{abstract}
This study describes the Computational Fluid Dynamics (CFD) analysis of the turbulent cross-flow in a staggered tube bundle with transverse and longitudinal pitch-to-diameter ratio of 3.8 and 2.1 respectively. Two Longitudinal grooves are placed on the external surface at $90^{\circ}$ and $270^{\circ}$ degrees. Each cylinder has two grooves on the external surface of the cylinder. The Navier-Stokes equations of the turbulent flow are solved using Spalart almaras, k- $\varepsilon$ realizable and k- $\omega$ SST, turbulence models provided by Fluent CFD code. The staggered tube bundle geometry simulations were performed at steady conditions. An adapted grid using static pressure, pressure coefficient and velocity gradient, furthermore, a second order upwind scheme were used. The CFD results were in agreement with the experimental data. The study demonstrates the capability of the CFD calculations in predicting flow characteristics around the bundle.
\end{abstract}

\section{Introduction}

Tube bundles are widely employed in cross-flow heat exchangers. The complex vortex shedding characteristics behind the cylindrical tubing can cause severe vibration of the tubes where serious structural failure is caused. Over the past years, numerous experimental investigations have been carried out to study the complex mechanism of the flow characteristics in different configurations of tube bundles. Romberg and Popp $([1,2])$ conducted experimental investigations on the stability factors of normal and rotated triangular tube arrays with different pitch-to-diameter ratios. Chen and Srikantiah [3] presented series of experiments to measure motion-dependent fluid forces for various tube arrays. Rottmann and Popp [4] further investigated the influence of approach flow on the first row of tube bundles. Paul et al. [5] preformed measurements in a staggered tube bundle with fixed pitch-to-diameter ratios at sub-critical Reynolds number using the particle image velocimetry technique. Their results showed that the flow exhibits strong Reynolds number dependence in the developing region, however no significant Reynolds number effect could be observed in the spatially periodic region.

Because of the complicated flow characteristics in the heat exchanger tube bundles, the vortex structures cannot be clearly observed and a lot of valuable information cannot be obtained by experimental techniques alone. It is then suggested that numerical simulations should be able to capture the instantaneous threedimensional vortex structures and other instantaneous fluid dynamic parameters, such as drag, lift, pressure, velocity and Reynolds stress. The first direct numerical simulations (DNS) in tube bundle flows appeared in the literature (Moulinec et al.[6]). The Reynolds number (based on the bulk velocity) was equal to 6000 and the finest mesh had $196 \times 196 \times 128$ cells. The computational domain comprised only an elemental (or periodic) 'cell', assuming fully developed flow in the streamwise and cross-stream directions. The predictions compared well with the experiments of Simonin and Barcouda [7] for both mean and r.m.s. velocities. DNS at various Reynolds numbers between 50 and 6000 and more elemental'cells' were also reported by Moulinec et al. [8]. Very interesting results on the wake disappearance were presented and compared with theoretical asymptotic limits for laminar and turbulent strained flows. However, information on vortex shedding was not reported in these papers.

The Large Eddy Simulation (LES) technique is situated between the RANS and DNS in the sense that large scales are calculated directly while the small ones are approximated. The fact that in tube bundles momentum and heat transfer are, to a large extent, controlled by large scale vortices shed behind the cylinders, makes this technique particularly attractive. The first simulations were two-dimensional (Hassan and Ibrahim, [9]; Barsamian and Hassan, [10]; Bouris and Bergeles, [11]; 
Hassan and Barsamian, [12]). However, the problem of two-dimensional LES calculations is that important mechanisms, such as vortex stretching, cannot be reproduced. Pioneering three-dimensional LES calculations inside a staggered tube bundle [studied experimentally by Simonin and Barcouda [7] were performed by Rollet-Miet et al. [13] and Benhamadouche and Laurence [14] using the finite element and finite volume methods, respectively. In both papers, the flow through an elemental 'cell' was simulated assuming periodicity in the streamwise and cross-stream directions and improved predictions compared to the RANS approach for mean and turbulence quantities were reported. They also observed that the subgrid-scale model, whether the standard Smagorinsky with constant coefficient or the dynamic version, had little effect on the results.

Although some interesting studies which are based on the premise of modified tube shapes have been carried out, most of the investigations have focused on the characteristics of fluidelastic instability of tube bundles with the circular cylinders. It was found that a cylinder with wavy surface can weaken, to the extent that it may even suppress the vibration comparing with a circular cylinder at the same Reynolds numbers (Lam et al., [15, 16]). An optimal spanwise wavelength ratio for drag reduction and fluctuating lift suppression was found at low Reynolds number of 100 over a wide wavelength range by Lam and Lin $[17,18]$.

The objective of the present study is to perform detailed assessment of the ability of the commercial CFD code, ANSYS Fluent, together with three different turbulence models Spalart almaras, k- $\varepsilon$ realizable and $\mathrm{k}-\omega \mathrm{SST}$ in their steady state formulations, to reproduce the mean flow and turbulent quantities in a staggered tube bundle. As is well known, turbulent flow in tube bundles is generally characterized by significant threedimensional flow structures and highly fluctuating wake regions with vortex shedding. Consistent with some of the previous studies, however, a two-dimensional steady state simplification has been chosen.

\section{Numerical modeling}

\subsection{Problem definition}

The geometry of the two-dimensional domain representing a typical region in a bundle with tube diameter " $D$ ", transverse pitch " $P$ ", and longitudinal pitch " $T$ " as shown in figure 1.the smooth cylinder are replaced by a grooved cylinder following the experiment of Ladjedel et al [19]. Fluid with an approach velocity, a velocity profile based on $U_{\infty}$, enters the solution domain instead of $U_{\infty}$ uniformly entered at the inlet region, a distance $L_{u s}=40^{*} \mathrm{D}$ from the centre of the first tube.

The raison of using a velocity profile is twofold:

-The location of inlet will not have a significant impact on the solutions obtained.
-To unburden the domain from additional cells (incorporate the $\mathrm{L}_{\mathrm{i}}$ in the geometry) that has a negative effect on the computational cost.

The outlet section is placed a distance $\mathrm{L}_{\mathrm{o}}$ from the centre of the last tube.

In the present study a numerical investigation of a crossflow in a staggered tube bundles has been performed. The Navier-Stokes equations of the turbulent flow are solved using Spalart-Allmaras, K- $\varepsilon$ realizable and k- $\omega$ SST turbulence models (RANS) provided by Fluent CFD code. The obtained results will be compared with the experiment of O.Ladjedel et al [20].

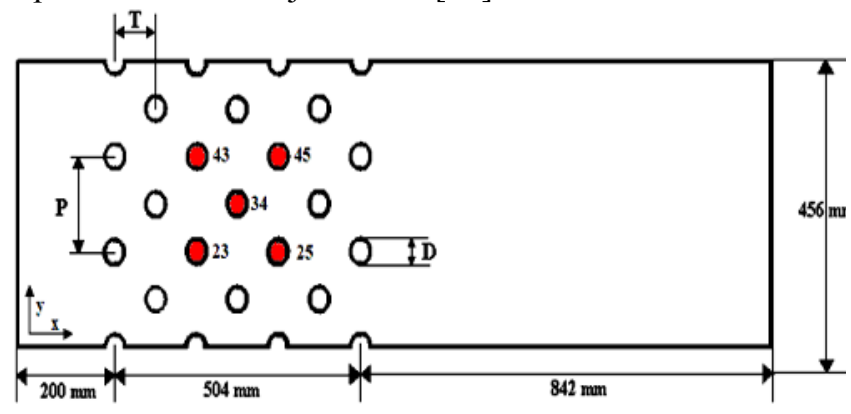

Fig. 1. Cross-sectional view of the tube bundle model with the definition of geometrical parameters.

\subsection{Mathematical formulation}

The flow is considered to be two-dimensional, incompressible, steady, and turbulent. The fluid is Newtonian with constant density $\rho$ and dynamic viscosity $\mu$ The Reynolds Averaged Navier-Stokes (RANS) equations for continuity and momentum conservation can be written as:

$$
\begin{gathered}
\frac{\partial U_{j}}{\partial x_{j}}=0 \\
\frac{\partial}{\partial x_{j}}\left(\rho U_{j} U_{i}\right)=\frac{\partial}{\partial x_{j}}\left(\mu \frac{\partial U_{i}}{\partial x_{j}}-\partial \overline{u_{j} u_{j}}\right)-\frac{\partial P}{\partial x_{i}}
\end{gathered}
$$

\subsection{Turbulence models}

The three turbulence models used in this study are one two two-equation model; $k-\varepsilon$ (Launder and Spalding 1994) and $k-\omega$ (Wilcox 1998), and one-equation model; Spalart-Allmaras.

\subsubsection{Spalart-Allmaras model}

$$
\frac{D \tilde{v}}{D t}=\frac{1}{\sigma \tilde{v}}\left[\frac{\partial}{\partial x_{j}}\left\{(\mu+\rho \tilde{v}) \frac{\partial \tilde{v}}{\partial x_{j}}\right\}+C_{b 2} \rho\left(\frac{\partial \tilde{v}}{\partial x_{j}}\right)\right]+G_{v}-Y_{v}+S_{\tilde{v}}
$$


Eddy viscosity is obtained from

$$
\mu_{t}=\rho \tilde{v f} f_{v 1}, \quad f_{v 1} \equiv \frac{(\tilde{v} / v)}{(v / v)^{3}+C_{v 1}^{\beta}}
$$

\section{Advantages}

-Economical for large meshes.

-Suitable for mildly complex (quasi-2D) external/internal flows and boundary layer flows under pressure gradient (e.g. airfoils, wings, airplane fuselage, missiles, ship hulls).

\section{Disadvantage}

-Perform poorly for 3D flows, free shear flows, strong separation

\subsubsection{Standard $k-\varepsilon$ model}

The k- $\epsilon$ model consists of the turbulent kinetic energy $(k)$ equation:

$$
\frac{D}{D t}(\rho k)=\frac{\partial}{\partial x_{j}}\left[\left(\mu+\frac{\mu_{t}}{\sigma_{k}}\right) \frac{\sigma k}{\sigma x_{j}}\right]+G_{k}-\rho \varepsilon
$$

And the dissipation rate $(\epsilon)$ equation:

$$
\frac{D}{D t}(\rho \varepsilon)=\frac{\partial}{\partial x_{j}}\left[\left(\mu+\frac{\mu_{t}}{\sigma_{\varepsilon}}\right) \frac{\sigma \varepsilon}{\sigma x_{j}}\right]+C_{e l} \frac{\varepsilon}{k} G_{k}-\rho C_{\varepsilon 2} \frac{\varepsilon^{2}}{k}
$$

$$
\text { Where } C_{\mu}=0.09, C_{\varepsilon 1}=1.44, C_{\varepsilon 2}=1.92, \sigma_{k}=1, \sigma_{\varepsilon}=1.3
$$

\section{Advantages}

-The most widely used in engineering turbulence model for industrial applications.

-Robust and reasonably accurate; it has many sub-models for compressibility, buoyancy, and combustion.

\section{Disadvantage}

-Performs poorly for flows with strong separation, large streamline curvature, and high pressure gradient.

\subsubsection{K- $\omega$ model}

$$
\mu^{t}=\alpha^{*} \rho \frac{k}{\omega}
$$

$\rho \frac{D k}{D t}=\tau_{i j} \frac{\partial U_{i}}{\partial x_{j}}-\rho \beta^{*} f_{\beta^{*}} k \omega+\frac{\partial}{\partial x_{j}}\left[\left(\mu+\frac{\mu_{t}}{\sigma_{k}}\right) \frac{\sigma k}{\sigma x_{j}}\right]$

$\rho \frac{D \omega}{D t}=\alpha \frac{\omega}{k} \tau_{i j} \frac{\partial U_{i}}{\partial x_{j}}-\rho \beta f_{\beta} k \omega^{2}+\frac{\partial}{\partial x_{j}}\left[\left(\mu+\frac{\mu_{t}}{\sigma_{\omega}}\right) \frac{\sigma \omega}{\sigma x_{j}}\right]$

Specific dissipation rate:

$$
\omega \approx \frac{\varepsilon}{k} \propto \frac{1}{\tau}
$$

\section{Advantage}

$-k-\omega$ can be integrated to the wall without using any damping functions.

-Accurate and robust for a wide range of boundary layer flows with pressure gradient and separation.

-Most widely adopted in the aerospace and turbomachinery communities.

-Several sub-models options of $\mathrm{k}-\omega$; compressibility effects, transitional flows and shear-flow corrections.

\subsection{Mesh-independence tests}

Several grids have been tested to investigate the influence of grid refinement on the solution; hence, three meshes were employed table 1. Figure. 3 shows a comparison between the experimental pressure coefficient distribution for the central tube of ref [20] and that of the three grids tested. It is clearly seen that there is a difference between the three grid results. The fine grid gives better results compared with the coarse and medium grids. Therefore the fine grid is used in all subsequent calculations

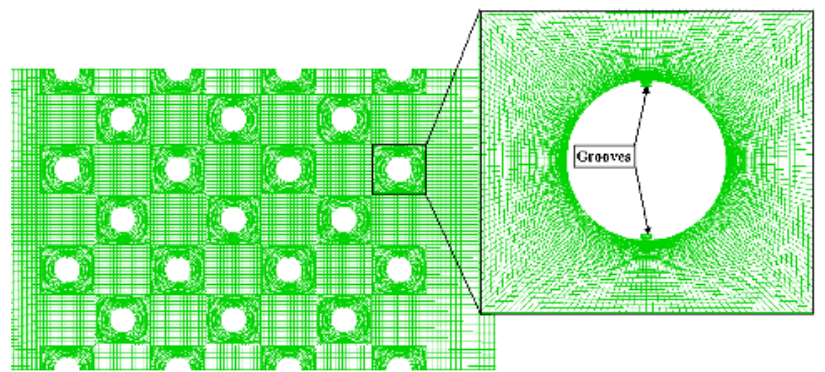

Fig. 2. Zoomed-in view of the computational mesh used in the simulation.

Table 1. Details of grids used in mesh-independence tests.

\begin{tabular}{|c|c|c|c|}
\hline Grid & Cells & Faces & Nodes \\
\hline A (Coarse) & 36558 & 74262 & 37688 \\
\hline B (Medium) & 108407 & 218699 & 110276 \\
\hline C (Fine) & 431412 & 866584 & 435156 \\
\hline
\end{tabular}

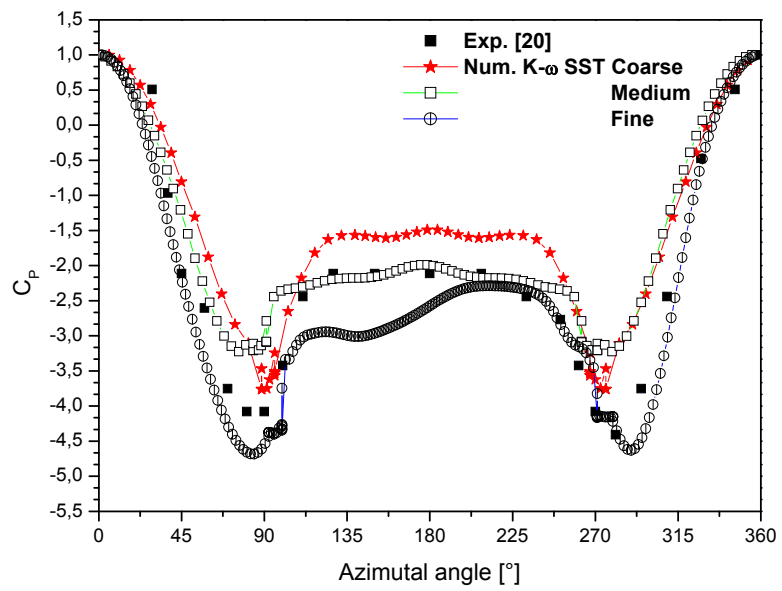

Fig.3. Mesh Independence Test 


\section{Results and discussion}

This numerical investigation is based on the following physical parameters:

- Reynolds number $=9300$ (based on free stream velocity).

- Free stream velocity: $U_{\infty}=3.5 \mathrm{~m} / \mathrm{s}$.

- Pitch ratio, $P=152 \mathrm{~mm}, T=84.0 \mathrm{~mm}$ and $\mathrm{D}=40 \mathrm{~mm}$.

Figure 4 shows a comparison between the three turbulence models tested and the experimental data of Ladjedel et al [20] for the central tube. The k-w sst model seems to follow perfectly the trend of the experiment allure compared with the two other models.

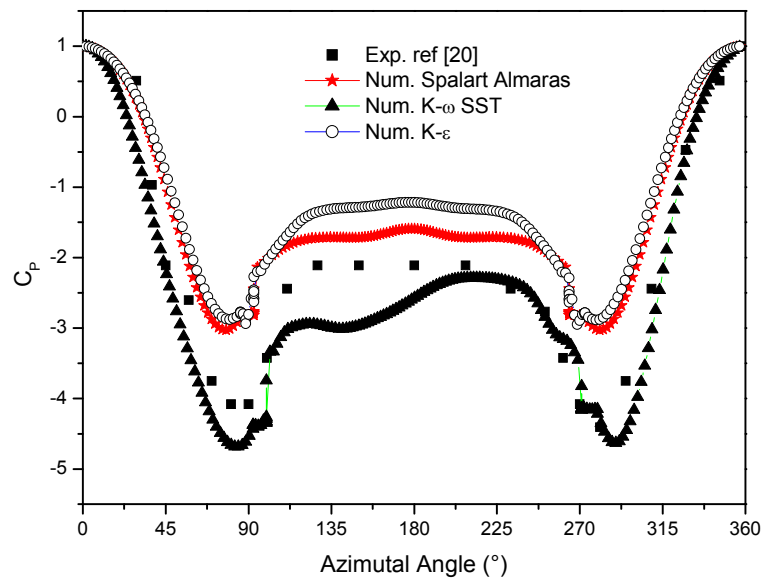

Fig.4. Mean pressure distribution around the centre tube, comparison between the 2D RANS models and experiment.

Figures 5, 6, 7 and 8 show the evolution of normalized pressure coefficient around the tube 2-3, 2-5, 4-3 and 4-5 respectively using the $\mathrm{k}-\mathrm{w}$ SST turbulence model. The trend is nearly the same for all the tested tubes. The obtained distributions seem to be similar to that of an isolated cylinder. It is notice that the stagnation point is located at an angle of $0^{\circ}$ and $360^{\circ}$ degree which correspond to the maximum pressure.

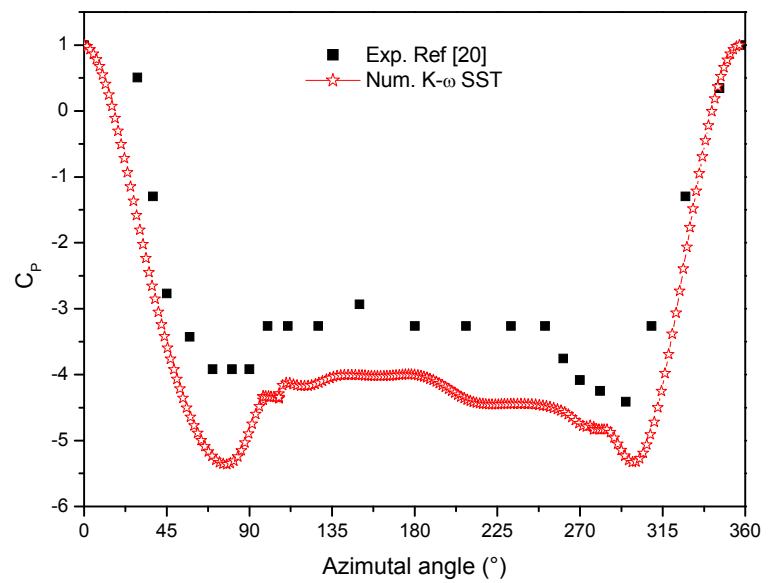

Fig. 5. Mean Pressure coefficient distribution for the tube 2-3.

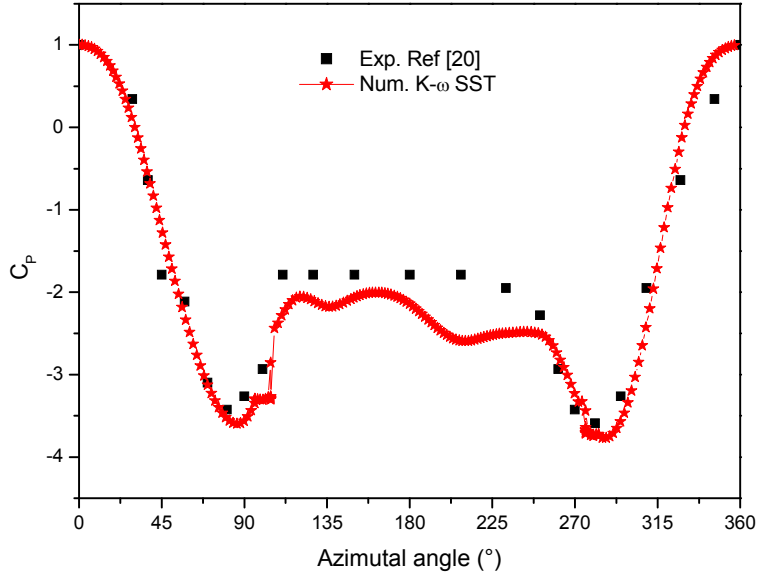

Fig. 6. Mean Pressure coefficient distribution for the tube 2-5.

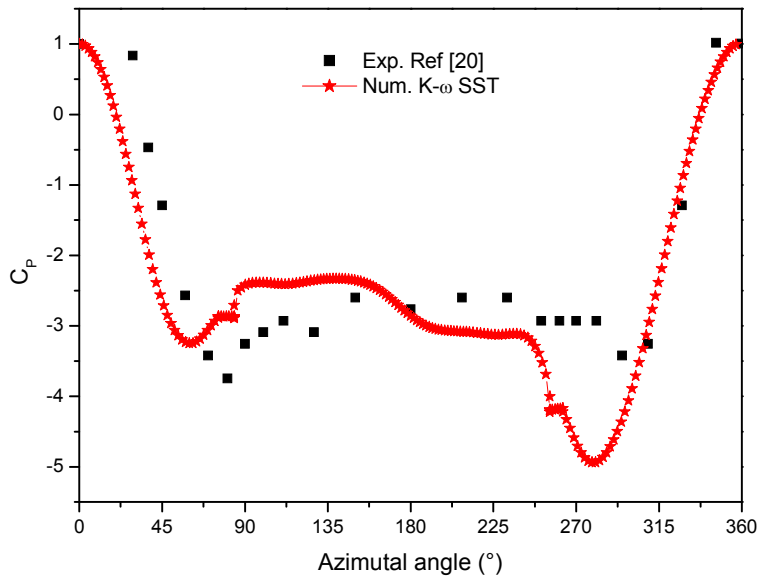

Fig.7. Mean Pressure coefficient distribution for 4-3.

The interaction between all the rows appears to be negligible. In the later region the curves seem to stay constants. Those results are in a good agreement with experimental data. However for the distributions of tubes 2-3 a little difference is noticed specially for the region discussed above where the flow is probably more disrupted in this region; hence it's difficult to predict it by the turbulence model.

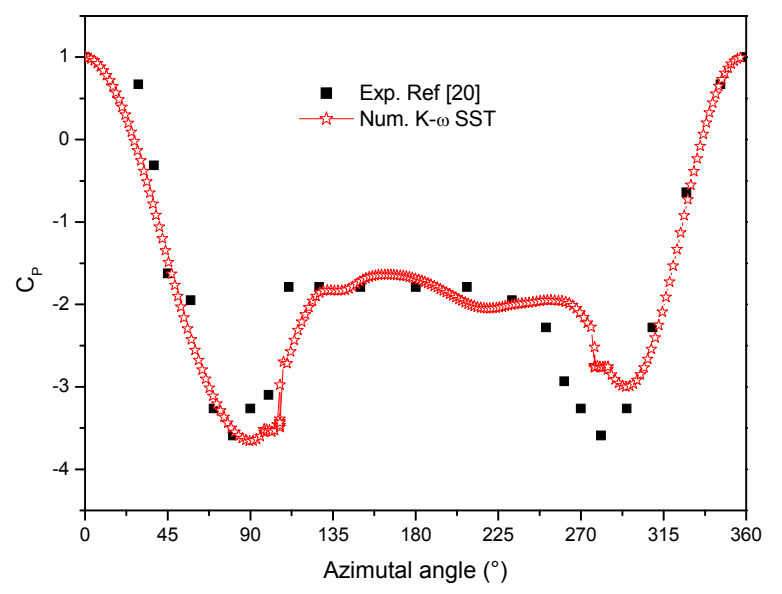

Fig. 8. Mean Pressure coefficient distribution for the tube4-5.

Snapshots of streamlines in $X Y$ plane for the entire configuration are shown in Figure. 9. Two recirculation bubbles coexist, a big one upstream the tube in the top 
due to the acceleration of the fluid and a small one on the bottom. The shear stress in the top of the tube is then higher than in the bottom.

Fig. 10 shows the streamlines generated from 2D mean velocity vectors field in $X Y$ plane for the studied cylinders. Over the tip of the cylinders the maximum velocity (which at the inlet is $3.5 \mathrm{~m} / \mathrm{s}$ ) goes as high as 10 $\mathrm{m} / \mathrm{s}$. One now observes that the shear layer separates from the bottom of the cylinder surface as well resulting in two recirculation regions behind every cylinder. However, the flow is still asymmetrical with one recirculation bubble considerably larger than the other. One observes from that the flow is still showing persistently asymmetric behaviour with flow going up between all rows of cylinders.

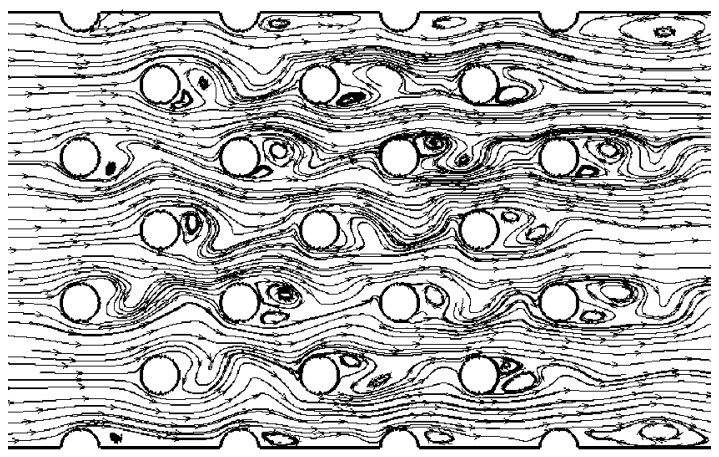

Fig. 9. Streamlines generated from 2D Mean velocity vectors in $X Y$ plane for the entire configuration (K-Omega SST).

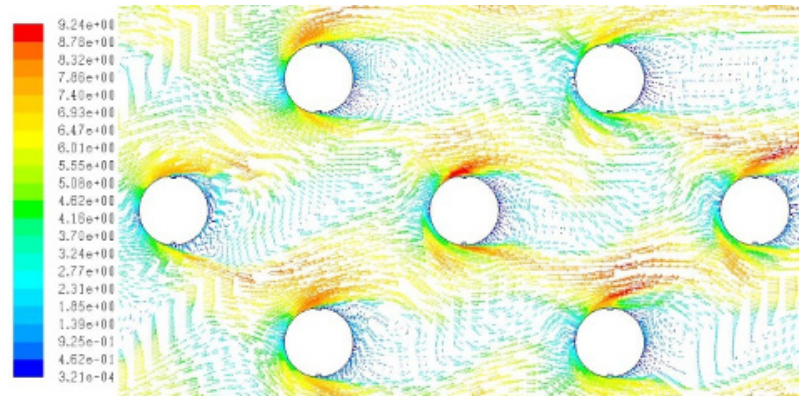

Fig. 10. Mean velocity vectors in $X Y$ plane for the studied tubes (K-Omega SST).

\section{Conclusion}

The following conclusions have to be drawn from the present work:

- The flow across in-line tube bundles is found to be symmetric and transient;

- RANS technique in 2D with RSM and approach is able to capture the transient flow behavior, while the $\mathrm{k}-\varepsilon$ model suppresses all flow fluctuations.

- Finally, good quantitative and qualitative agreement with the experimental results shows the ability of the present approach (RANS) to correctly reproduce the essential physics associated with massively separated flow.

\section{Reference}

1. Romberg, O., Popp, K. The influence of trip-wires on the fluid dampingcontrolled instability of a flexible tube in a bundle. J. Fluid Struct. 12, 17-32 (1998a)

2. Romberg, O., Popp, K. The influence of upstream turbulence on the stability boundaries of a flexible tube in a bundle. J. Fluid Struct. 12, 153-169 (1998a)

3. Chen, S.S., Srikantiah, G.S. Motion-dependent fluid force coefficients for tube arrays in crossflow. ASME J. Press. Vess. Technol. 123, 429-436 (2001).

4. Rottmann, M., Popp, K. Influence of upstream turbulence on the fluidelastic instability of a parallel triangular tube bundle. J. Fluid Struct. 18, 595-612 (2003)

5. Paul, S.S., Tachie, M.F., Ormiston, S.J. Experimental study of turbulent crossflow in a staggered tube bundle using particle image velocimetry. Int. J. Heat Fluid Flow 28, 441-453 (2007).

6. Moulinec, C., Pourquie' , M.J.B., Boersma, B.J., Buchal, T., Nieuwstadt, F.T.M. Direct numerical simulation on a Cartesian mesh of the flow through a tube bundle. International Journal of Computational Fluid Dynamics 18, 1-14 (2004b)

7. Simonin, O., Barcouda, M. Measurements and prediction of turbulent flow entering a staggered tube bundle. In: Proceedings of Fourth International Symposium on Applications of Laser Anemometry to Fluid Mechanics, Lisbon, paper number $\mathbf{5 . 2 3}$ (1988)

8. Moulinec, C., Hunt, J.C.R., Nieuwstadt, F.T.M. Disappearing wakes and dispersion in numerically simulated flows through tube bundles. Flow, Turbulence and Combustion 73, 95-116 (2004a)

9. Hassan, Y.A., Ibrahim, W.A. Turbulence prediction in two-dimensional bundle flows using large eddy simulation. Nuclear Technology Journal 119, 11-28 (1997)

10. Hassan, Y.A., Barsamian, H.R. Tube bundle flows with the large eddy simulation technique in curvilinear coordinates. International Journal of Heat and Mass Transfer 47, 3057-3071 (2004)

11. Bouris, D., Bergeles, G. Two dimensional time dependent simulation of the subcritical flow in a staggered tube bundle using a subgrid scale model. International Journal of Heat and Fluid Flow 20, 105-114 (1999)

12. Hassan, Y.A., Barsamian, H.R. Turbulence simulation in tube bundle geometries using the dynamic subgrid-scale model. Nuclear Technology Journal 128, 58-74 (1999)

13. Rollet-Miet, P., Laurence, D., Ferziger, J.H. LES and RANS of turbulent flow in tube bundles. International Journal of Heat and Fluid Flow 20, 241-254 (1999)

14. Benhamadouche, S., Laurence, D. LES coarse LES, and transient RANS comparisons on the flow across a tube bundle.International Journal of Heat and Fluid Flow 24, 470-479 (2003) 
15. Lam, K., Wang, F.H., Li, J.Y., So, R.M.C. Experimental investigation of the mean and fluctuating forces of wavy (varicose) cylinders in a cross-flow. J. Fluid.Struct. 19, 321-334 (2004a).

16. Lam, K., Wang, F.H., So, R.M.C. Three-dimensional nature of vortices in the near wake of a wavy cylinder. J. Fluid Struct. 19, 815-833 (2004b).

17. Lam, K., Lin, Y.F. Drag force control of flow over wavy cylinders at low Reynolds number. J. Mech. Sci. Technol. 21, 1331-1337 (2007).

18. Lam, K., Lin, Y.F. Effects of wavelength and amplitude of a wavy cylinder in cross-flow at low Reynolds numbers. J. Fluid Mech. 620, 195-220 (2009).

19. O. Ladjedel, T.Yahiaoui, L.Adjlout and O.Imine 2011. Experimental and Numerical Studies of Drag Reduction on a Circular Cylinder. World Academy of Science, Engineering and Technology v 77 P: 357-361.

20. O. Ladjedel, T.Yahiaoui, L.Adjlout and O.Imine. Experimental investigation on staggered tube bundle arrangement using grooved cylinder. Internal report Marine Engineering Department, Mechanical Engineering Faculty USTO Oran, Algeria 2012. 